\title{
Making decisions in the management of perinatal depression and anxiety
}

\author{
Anne P. F. Wand
}

\begin{abstract}
SUMMARY
The care of women with anxiety and depressive disorders in the perinatal period is complex. The literature in this field is vast and may be difficult for busy clinicians to keep abreast of. The first part of this article provides an overview of the potential risks and benefits of treatment options, including no treatment, at various stages in the perinatal period. The second part explores the frameworks which may assist clinicians in decision-making with their pregnant patients, including risk-benefit analysis, ethical considerations, evaluating capacity, and mental health legislation. The common pitfalls and limitations of these approaches are examined to guide good practice.
\end{abstract}

\section{LEARNING OBJECTIVES}

- Understand the potential risks and benefits of treating, or not treating, maternal mental illness at various stages in the perinatal period.

- Understand the limitations of the literature in this field.

- Be able to use different frameworks for deciding with patients about the management of mental illness in the perinatal period.

\section{DECLARATION OF INTEREST}

None.

The mental healthcare of a pregnant or postpartum woman is frequently a part of psychiatric practice. Estimates of the prevalence of depression in pregnancy range from 3 to $14 \%$, depending on severity and how the data are collected (Chaudron 2013). Rates of anxiety disorders and pregnancyrelated anxiety are even higher (Ross 2006). To best inform their patients, clinicians need to be aware of the potential risks and benefits of treatment and of opting for no treatment at various stages in the perinatal period, taking into account the mother, fetus and existing children. Detailed evidence-based clinical guidelines for perinatal mental illness are available from, for example, Beyondblue (2011), the National Institute for Health and Care Excellence (National Institute for Health and Clinical Excellence 2007) and the Scottish Intercollegiate Guidelines Network (2002). A synopsis of the issues is presented here.

\section{Considerations during the perinatal period}

Maternal health in pregnancy

Untreated antenatal major depression adversely affects maternal health. Depressive symptoms in pregnancy are associated with poor nutrition (Zuckerman 1989), cigarette smoking (Zuckerman 1989; Marcus 2003; Goedhart 2010), increased alcohol intake (Zuckerman 1989; Marcus 2003) and pre-eclampsia (Kurki 2000). Maternal depression is also associated with poor weight gain and decreased social support (Zuckerman 1989). Maternal health may also be compromised by the social, occupational and physical role impairment associated with depression (Wisner 2000). Additionally, the symptoms of depression, such as fatigue, suicidal ideation, anxiety, pervasively low mood and guilt, may in themselves be distressing. Antenatal depression predisposes the mother to postnatal depression (Pearlstein 2008).

\section{Obstetric and neonatal outcomes}

\section{Depression in pregnancy}

Treatment of depression in pregnancy, as with onset at any time, may include pharmacotherapy, electroconvulsive therapy (ECT) and psychotherapy. Lifestyle changes such as avoiding drug and alcohol use, taking up exercise and building social networks are also important, as is providing ongoing support and monitoring (Dimidjian 2009).

Pharmacotherapy There have been inconsistent findings regarding associations between antidepressants (mainly selective serotonin reuptake inhibitors (SSRIs) and serotonin-noradrenaline reuptake inhibitors (SNRIs)) and spontaneous abortion, prematurity, small for gestational age, persistent pulmonary hypertension of the newborn, and low birth weight (see Galbally 2011) (Box 1). A well-designed Danish register study of all pregnancies from 1996 to 2006 found increased rates of low Apgar scores ( $<7$ at $5 \mathrm{~min}$ ) in infants exposed to SSRIs in pregnancy, but no association with maternal depression without antidepressant prescription before or during pregnancy, or use of newer antidepressants or tricyclic antidepressants (TCAs) (Jensen 2013).
Anne Wand is a conjoint senior lecturer with the University of New South Wales, a clinical lecturer with the University of Sydney, and a staff specialist psychiatrist in South Eastern Sydney Local Health District in Sydney, Australia. Her research interests include perinatal and migrant mental health, decisionmaking capacity and other legal aspects of psychiatry, and delirium. Correspondence Dr Anne Wand, South Eastern Sydney Local Health District, c/o Department of Consultation Liaison Psychiatry, Prince of Wales Hospital, Euroa Centre, Barker St, Randwick, New South Wales 2139, Australia. Email: anne.wand@sydney.edu.au 
BOX 1 Main risks of antidepressant medications in pregnancy

Maternal

- Side-effects of SSRIs (e.g. gastrointestinal symptoms, headache), TCAs (e.g. sedation, constipation, weight gain)

Fetus/child

- Conflicting evidence (positive and negative studies) for spontaneous abortion, small for gestational age persistent pulmonary hypertension of the newborn, preterm delivery, low birth weight

- Low Apgar scores (SSRIs)

- If combined with benzodiazepines, there are higher rates of congenital defects

- Neonatal serotonin discontinuation syndrome

- Development: impaired fine motor skills in children

A recent large cohort-based study from all Nordic countries over a 10 -year period found no significant association between the use of SSRIs in pregnancy (for any indication) and stillbirth or neonatal mortality, when maternal characteristics and severity of psychiatric illness were controlled for (Stephansson 2013). However, there is more consistent evidence for neonatal serotonin discontinuation syndrome with antenatal antidepressant use (Galbally 2011). The syndrome is characterised by gastrointestinal, central nervous system and respiratory symptoms (Galbally 2011). The syndrome is usually brief and self-limiting.

One of the issues complicating the interpretation of the literature is that concurrent medication use during pregnancy or health habits (e.g. smoking) may confound possible associations (Yonkers 2009). For example, SSRIs taken with benzodiazepines, but not SSRIs alone, may be associated with higher rates of congenital health defects compared with no exposure, even when the severity of illness is controlled for (Oberlander 2008). An additional consideration is that few studies separate mothers with depression into medicated or unmedicated groups (Grote 2010), or monitor for medication adherence. This is important to tease out, as higher rates of preterm delivery (Suri 2007) and increased risk of low birth weight and respiratory distress (Oberlander 2006) have been reported with antenatal antidepressant use, but not unmedicated depression in some studies.

ECT Electroconvulsive therapy has been used to treat depression in pregnancy, particularly when the illness is not responsive to antidepressants or other treatments, or when there are lifethreatening symptoms such as suicidal ideation, poor oral intake, catatonia or psychosis.

Anderson $\&$ Reti (2009), in a literature review, found at least partial response to ECT in $84 \%$ of pregnant women with depression. Of the 339 published cases of ECT in pregnancy they reviewed, fetal or neonatal abnormalities were reported in 25 cases, but only 11 of these were likely related to ECT. These adverse effects included transient fetal arrhythmia $(n=8)$, fetal death $(n=2$; one secondary to status epilepticus and another was a first trimester miscarriage) and 1 case of multiple cerebral infarction (after multiple ECT courses in pregnancy). The 18 maternal cases of adverse effects from ECT included uterine contraction/ preterm labour, vaginal bleeding, abdominal pain, placental abruption, status epilepticus and haematuria (Anderson 2009). Four of the included cases overlapped with both neonatal and maternal complications.

Adverse effects may be reduced by ensuring adequate preoxygenation, positioning the mother to minimise aortocaval compression, monitoring obstetric fetal movements and tocodynamometry (for uterine contractions), and using low doses of anaesthetics (Anderson 2009).

Psychotherapy There is a dearth of evidence regarding the efficacy of non-pharmacological treatment of antenatal depression (Dimidjian 2009). A meta-analysis of treatments for depression in the perinatal period by Bledsoe $\mathcal{E}$ Grote (2006) included 13 studies of psychotherapy without medication (interpersonal therapy (IPT), cognitive-behavioural therapy (CBT), group therapy, counselling, education and psychodynamic therapy). The authors found the largest effect sizes $(>0.95)$ for CBT, group therapy with aspects of CBT, educational and transactional analysis, and IPT. They noted that several of the treatment types were represented by single studies only.

Barriers to women accessing psychotherapy have been noted, including the financial cost, time commitment, lack of available trained therapists and difficulties with child care (Pearlstein 2008). Given the preference of many women for non-pharmacological treatment in pregnancy (Goodman 2009), this is a significant matter to be addressed.

Untreated depression There are few studies exploring the potential adverse outcomes associated with untreated maternal depression on the fetus (Chaudron 2013) (Box 2). This is in part due to ethical concerns in conducting such 
studies. Most results are inconclusive, with a mix of positive and negative studies. It is unclear whether untreated depression is associated with miscarriage, as studies are limited by small sample sizes and methodological problems (Yonkers 2009). Antenatal depression has been associated with low Apgar scores (Goedhart 2010) and babies small for gestational age in some (Oberlander 2006) but not all (Yonkers 2009) studies. Low birth weight and increased risk of preterm birth have also been associated with antenatal depression in some studies, but effect sizes are relatively small (Grote 2010). When compared directly, SSRI use or untreated major depression were both associated with higher rates of preterm delivery (Wisner 2009). Not all studies have found an association between antenatal depression and growth-restricted or small for gestational age fetuses or preterm birth (see Goedhart 2010).

In contrast to the numerous studies of antidepressants in pregnancy, to date there are no studies evaluating whether untreated depression is associated with structural malformations in the fetus (Chaudron 2013).

\section{Anxiety disorders}

Maternal anxiety during pregnancy has also been associated with adverse obstetric and neonatal outcomes (Box 2). Pregnancy anxiety, or anxiety regarding a current pregnancy, has been fairly consistently associated with shorter gestation and preterm birth, in rates comparable to other known risk factors such as medical and obstetric risks and smoking (Dunkel Schetter 2012). Pregnancy anxiety has also been associated with higher rates of emergency Caesarean sections in one study (Ryding 1998) but not others (Reck 2013), and longer duration of labour (Reck 2013).

There are very few studies examining the relationship between actual anxiety disorders and perinatal outcomes. One well-designed study did not find associations between adverse perinatal outcomes and generalised anxiety disorder, panic disorder, social phobia or obsessive-compulsive disorder during pregnancy (Andersson 2004). Another did demonstrate an association between post-traumatic stress disorder and adverse outcomes such as spontaneous abortion and excessive fetal growth, although notably could not control for medication use in women with posttraumatic stress disorder (Seng 2001).

The pharmacological treatment of anxiety disorders includes antidepressants (e.g. SSRIs, SNRIs, TCAs) and benzodiazepines. The potential risks associated with taking these medications in pregnancy are discussed earlier.
BOX 2 Main risks of untreated depression and anxiety in the perinatal period
Maternal

- Poor maternal health (nutrition, poor weight gain) (depression)

- Increased rates of maternal smoking and alcohol use (depression)

- Pre-eclampsia (depression)

- Conflicting evidence for increased rates of emergency Caesarean section (anxiety)

- Longer duration of labour (anxiety)

- Suicidal ideation, distress from symptoms

Fetus/child

- Conflicting evidence for small for gestational age and low Apgar scores (depression)

- Low birth weight (small effect size for depression) and role impairment
- Shorter gestation and preterm birth (anxiety; small effect size for depression)

- Cognition: reduced sustained attention and self-regulation in adolescents (anxiety); lower IO in adolescents (postnatal depression)

- Development: poorer motor and language delay in 18-month-olds (antenatal interactions (postnatal depression)

- Psychopathology in children: behavioural and emotional problems, attentiondeficit hyperactivity disorder symptoms, externalising problems, self-reported anxiety (antenatal anxiety); internalising and externalising problems (antenatal depression) skills in toddlers (anxiety); developmental depression); impaired mother-infant

\section{Childhood outcomes}

\section{Childhood development}

There is mounting research concerning the childhood developmental risks associated with antenatal depression and anxiety and their pharmacological treatment (Box 2). It is difficult to interpret these studies as few distinguish between the timing of exposure to maternal mental illness, differentiate anxiety and depression or their combination, or consider maternal antenatal antidepressant or other medication use (and factors such as duration of use and adherence), and lifetime maternal depression. One review concluded that in relation to perinatal exposure to maternal depression or maternal anxiety and stress, antenatal exposure to maternal anxiety and stress had the most consistent and greatest negative effects on child and adolescent outcomes (Brand 2009). These effects included reduced sustained attention and selfregulation in 15-year-olds and poorer cognitive/ motor skills and receptive/expressive verbal language in toddlers (summarised in Brand 2009). A large prospective study found an association between antenatal depression and developmental delay in 18-month-olds (Deave 2008).

There is one published study with contradictory findings. DiPietro and colleagues (2006) found that maternal anxiety and depression were associated with higher motor and mental scores in 2-year-olds. Brand \& Brennan (2009) though, suggest that these unexpected findings may be 
attributable to the population studied (nonclinical, high-functioning pregnant women).

A recent review concluded that there was limited information on long-term growth, intelligence and behavioural outcomes in children exposed to SSRIs or TCAs antenatally (Chaudron 2013), although a few studies have demonstrated impairment of fine motor skills in children who are exposed (Gentile 2011). A comparison of fluoxetine, TCAs and controls did not demonstrate any difference in IQ, language development or behavioural development in toddlers and children aged 5-6 years (Nulman 1997). However, depression duration was negatively associated with IQ and the number of maternal depressive episodes after delivery was negatively associated with language in a comparison of fluoxetine, TCAs and controls (Nulman 2002).

\section{Child behaviour and psychopathology}

Antenatal anxiety Antenatal anxiety, stress and depression may also have effects on child behaviour and psychopathology (Box 2). Stress and anxiety in pregnancy are associated with more behavioural and emotional problems in children, in particular attention-deficit hyperactivity disorder symptoms, externalising problems and self-reported anxiety in children (Brand 2009). The effect in relation to symptoms of impulsivity and hyperarousal appears to be greatest in boys.

Antenatal depression Antenatal depression has been associated with internalising and externalising problems in children (Brand 2009). A recent population-based case-control study in Sweden found that a history of maternal depression was associated with a greater risk of autism spectrum disorder without intellectual disability in children (Rai 2013). The association was limited to women reporting antenatal use of antidepressants. The severity and course of depressive symptoms was not known. However, as depression was identified using specialist psychiatric care records, this finding may reflect severe depression rather than an exclusive effect of medication use (Rai 2013). Maternal distress, i.e. subclinical psychological and psychosomatic symptoms, in early childhood may predict pre-adolescent depression in girls (Pitzer 2011).

Postnatal depression Postnatal depression may impair the interaction between the mother and baby and adversely affect the child's development. For example, mothers with depression may lack normal verbal and non-verbal (including facial) communication, be unresponsive or insensitive to their infant's needs and communication, be deficient in touching their child, and engage in activities inappropriate for the child's developmental stage or temperament (Punamäki 2006). Maternal postpartum (but not antenatal) depression has also been associated with lower adolescent IQ, especially in males (Hays 2008).

However, it is not just the perinatal period that is important. It appears that ongoing exposure to maternal depression during infancy and early childhood more strongly predicts child outcomes than exposure to depression in the postpartum period (Halligan 2007).

\section{Approaches to decision-making}

Given the complexity and breadth of information available and the gaps in knowledge regarding the treatment of anxiety and depression in the perinatal period, it is important for clinicians to have a framework for these discussions with their patients. Clinical decision-making may be guided by the physician's own values and experiences as well as patient preference. However, decisions about treating mental illness in the perinatal period are also made within social, ethical and legal contexts. Exploration of these factors may assist the clinician in making well-balanced treatment decisions with their patients (Box 3).

\section{Risk-benefit approach}

In modern Western societies there has been greater emphasis on the empowerment of consumers in making decisions about their healthcare. The most

BOX 3 Considerations associated with frameworks guiding decision-making

- Risk-benefit analysis: including background risk of malformations, accurate appraisals of teratogenicity, equal weight given to the potential outcomes of opting not to treat or of discontinuing effective treatment, evidence-based treatment options

- Capacity and ethics: autonomy and beneficence, relational ethics (i.e. the well-being of the mother and baby are interrelated), influence of patient's depression and anxiety symptoms on her decision-making capacity and the clinician, omission bias (unequal emphasis placed on the risk of active treatment compared with that of not treating), measures to enhance decisionmaking capacity (non-directive counselling, substitute decision maker), societal pressure on mothers

- Mental health legislation: risk of harm to the mother or others from the mental illness

- Child protection issues: especially when there are existing children 
commonly espoused approach to making decisions about the treatment of mental health problems in pregnancy is risk-benefit analysis. This approach involves the clinician outlining the problem or diagnosis, then discussing the potential risks and benefits to the mother and fetus/baby of various treatment options (Wisner 2000).

The choices an individual woman makes may change depending on her stage in the perinatal period, the corresponding risk and benefits of treatment, and her circumstances and symptoms (Chaudron 2013). As summarised in the preceding section, because depression and anxiety may influence outcomes for the mother and fetus/baby, the discussion should also incorporate the risks and benefits of not treating the illness (Yonkers 2009). The woman, presumed competent, is then encouraged to make a decision in line with her own values. The process is ongoing and requires further exploration as the pregnancy continues and responses to treatment emerge (Wisner 2000).

\section{Teratogenic risks}

Birth defects occur naturally in a significant number of neonates without exposure to medication in utero (Ramos 2007). Therefore, in discussions with women about the potential risks of using psychotropics in pregnancy, the background prevalence of major malformations (1.5-3\%; International Clearinghouse for Birth Defects Monitoring Systems 2003) should also be presented. Doctors and pregnant women in particular are inclined to overestimate the teratogenic risk of using medication in pregnancy (Sanz 2001). For example, the teratogenic risk of benzodiazepines was perceived to be $9.8 \%$ by physicians and $64.6 \%$ by pregnant women, although the actual risk was $<5 \%$ (Sanz 2001). This is of concern as pregnant women may be denied the benefits of relatively safe medication or be reluctant to take a recommended medication due to an excessively high perception of risk (Sanz 2001).

As a result of such misperceptions, many women decide to suddenly stop their antidepressants when they discover they are pregnant, often with their physician's recommendation or support. This is particularly relevant given significantly higher rates of relapse of depression on discontinuation of antidepressants in pregnancy (Cohen 2006).

\section{Suboptimal medical regimens}

The exaggerated perception of risk may lead physicians and patients to discount the potential value of pharmacotherapy, by discontinuing important medications at times of significant risk of relapse or by using suboptimal medication regimens. In one study, two-thirds of pregnant women felt that it was unacceptable to take antidepressants in pregnancy, but only a third thought it would be unacceptable if they were not pregnant or were breastfeeding (Goodman 2009). It has been observed that antidepressant use tends to progressively decrease from the first (3.7\%) to third (1.1\%) trimester (Ververs 2006). This trend has been attributed to patient and clinician concern about the link between exposure to antidepressants late in pregnancy and poor neonatal adaptation (Chaudron 2013).

An additional concern is the undertreatment of depression in pregnancy, with reductions in the dosage of antidepressants to subtherapeutic levels in $8 \%$ of women in one study (Ramos 2007). This is despite the fact that the doses of some antidepressants must be increased later in pregnancy to maintain euthymia due to changes in maternal physiology. The reason for the use of lower dosages in pregnancy is unclear and no studies have yet demonstrated a dose-dependent relationship between exposure to antidepressants and outcomes for mother or baby (Chaudron 2013).

\section{Ethics and capacity}

It has been argued that psychiatrists have an ethical responsibility to assess the degree of autonomy in pregnant women with depression, as it may be impaired by the illness (Coverdale 1997). Ethical issues in the perinatal period require the clinician to consider the needs of the mother as well as her fetus/baby (Miller 2009). Decisionmaking may become complicated when it appears that there is conflict between the well-being of the mother and that of the fetus/baby, for example when a mother's refusal to accept treatment for a mental illness could potentially harm the developing fetus. In this situation the clinician is torn between respecting the mother's autonomy (to make her own decision in line with her beliefs, values and priorities) and acting with beneficence (acting in the patient's best interest) (Miller 2009).

\section{Relational ethics}

Relational ethics has been proposed as a means of guiding decision-making when the above situation arises (Miller 2009). The emphasis of relational ethics is that the well-being of the mother and baby are intertwined - 'the maternal/fetal unit' - rather than separate (Seeman 2004). Seeman (2004) argues that under this ethical framework, safety concerns for the mother/fetal unit should always take precedence over maternal autonomy alone. 
Miller (2009) discusses a case vignette of a pregnant woman with bipolar disorder who acts on delusional beliefs and attempts to kill her fetus. Miller points out that the mother's own mental health is likely to deteriorate if she harms her fetus, and therefore that treating the mother's illness despite her refusal of treatment may be justified as much for the mother's well-being as for that of the fetus. Of course, there is also the issue of whether the mother is actually able to make an autonomous decision given the influence of her psychotic symptoms (Miller 2009).

\section{Major depression}

Major depression may also limit decisionmaking capacity, as although the individual may understand the facts presented to them about admission to hospital or treatment, their decisionmaking may be influenced by negative thinking, hopelessness or negative self-appraisals such that they are unable to weigh those facts in the balance to come to a decision (Coverdale 1997; Callaghan 2012).

Miller (2009) provides another case vignette of a woman with a history of major depression, who discontinues medication while trying to conceive and then relapses while pregnant. The woman declines to recommence medication for fear of harming the fetus, but raises concerns of being an inadequate mother and wanting to give the baby up for adoption, then kill herself. In this case the mother can articulate the risks to her baby of exposure to antidepressants as well as the risk to herself of remaining off medication, but she discounts the latter as irrelevant. The ethical conflict of patient autonomy over beneficence is again apparent. Miller (2009) suggests that even though reality-testing and cognition are intact, the symptoms of major depression may lead a mother to exaggerate the significance of risks to her fetus and devalue herself.

\section{Substitute and supported decision-making}

When depression significantly compromises decision-making, a substitute decision maker may be needed and a beneficence-based approach may be more relevant (Miller 2009). Another approach to this ethical dilemma is for the clinician to enhance the woman's autonomy by assisting her to identify her values for the pregnancy (Coverdale 1997). The clinician asks the patient what is important to her (now and prior to illness) about the pregnancy, the fetus and her own health. The patient could then be educated about her decisionmaking and how the depressive symptoms may influence this process. In this approach of 'non-directive counselling' the various alternatives are discussed, but no particular course of action is recommended by the clinician (Coverdale 1997). Involvement of the woman's spouse or family in the evaluation of her decision-making ability may also enhance her autonomy, particularly in assessing the influence of depressive symptoms on prior values and wishes.

\section{Risk perception is influenced by how information is presented}

Objective information (risk estimates) and subjective interpretation (the importance to the individual of various possible negative outcomes) are combined when making judgements about risk (Bogardus 1999). The way in which the information is presented can alter risk perception, which in turn influences decision-making (Wisner 2000). Thus, clinicians must be mindful to provide accurate information to patients (including the nature, degree and probability of risks of treatment and depression itself) that they can understand, despite the patient's fear of potential adverse outcomes.

Clinicians may inadvertently collude with an anxious mother's excessive fear about harming her fetus or depressive symptoms such as hopelessness or feeling overwhelmed, and opt not to recommend treatment in the perinatal period. It has been noted that societal 'moral standards of sacrifice' expected of mothers are already high and that pregnancy amplifies these expectations (Lyerly 2009). This may contribute to a tendency to overemphasise and weigh any risk to the fetus, no matter how remote or unclear, over considerations for the mother's well-being (Lyerly 2009).

\section{Omission bias}

A related consideration is whether clinicians, in obtaining informed consent for treatment of a mental illness, place equal emphasis on the risks of treatment and of untreated mental illness. That is, was the clinician equally specific, clear and detailed in their discussion of the risk of untreated maternal depression on the fetus as they were in describing the potential risks of antidepressants (Miller 2009)? This is known as omission bias.

In general, studies of medical decision-making reveal that physicians worry more about risks of commission (actively doing something such as a procedure or prescribing medication) than of omission (not doing something) (Kordes-de Vaal 1996). The underlying reason for this appears to be that physicians perceive themselves as more responsible for causing harm if the harm results from something they do, compared with something 
they do not do (Kordes-de Vaal 1996). As the causal link between an omission and its consequences is perceived as less strong, an omission may be viewed as a less intended (or non-) decision than a commission (Kordes-de Vaal 1996). Nonetheless, patients are not able to make accurate informed decisions when information is presented in a biased way. To prevent omission bias, clinicians should present the risks of withholding treatment as well as the risks of treatment (Miller 2009).

\section{Mental health laws - general considerations}

Clinicians may also look to the mental health act in their jurisdiction to enable the treatment of mental illness in the perinatal period, especially when the patient is very unwell and has declined treatment. The criteria required to detain and enforce treatment of a mental illness vary between states, territories and countries. It is beyond the scope of this article to examine international variations in pertinent mental health law. For the purpose of illustration, in Australia and New Zealand the requirements for coercive treatment under all mental health acts are that the individual has a mental illness (as defined under the act) and is considered likely to harm themselves or to harm others (Callaghan 2012). In Australia, the law is clear that the fetus does not constitute a legal person ( $R$ v Iby [2005]). The mother has the right to autonomy over her body, which includes during pregnancy (St George's Healthcare NHS Trust v S; $R$ v Collins and others, ex parte $S$ [1998]).

Although the fetus is not considered a legal person and therefore may not be a factor considered when evaluating the potential risks conferred by the mother's untreated mental illness, adverse outcomes for the fetus may affect the mother (see earlier), and the welfare of existing children certainly is legally relevant. As outlined previously, children may develop behavioural and emotional problems or a developmental disability as a result of their mother's untreated mental illness. This scenario falls clearly under the jurisdiction of child protection services. Removal of the children from the care of their mother may be a legitimate alternative. It is unclear whether this is in the best interests of both the mother and the children, or whether such an action would be perceived as more distressing than simply treating the mother's mental illness.

A related issue is the concerning emergent practice of criminalising substance misuse during pregnancy, when it is thought to be associated with adverse effects on the fetus (American College of Obstetricians and Gynecologists 2011). In several US states, substance misuse in pregnancy is considered child abuse or neglect under civil child welfare law and in some jurisdictions is grounds for involuntary internment in a substance misuse treatment facility or mental health unit (American College of Obstetricians and Gynecologists 2011). This practice has occurred despite evidence that incarceration or threat of incarceration are ineffective in reducing the incidence of substance misuse and may deter women from seeking antenatal care (the latter greatly mitigates the negative effects of substance misuse in pregnancy) (American College of Obstetricians and Gynecologists 2011). This is an extreme example of denial of maternal autonomy and places the clinician in a conflicted position as both treating doctor and mandatory reporter (American College of Obstetricians and Gynecologists 2008).

\section{Conclusions}

The literature concerning the potential risks and benefits of treatment at various time points in the perinatal period is considerable and at times conflicting, and may thus be difficult to interpret and explain to patients. Clinical decisions need to place equal emphasis on the risks and benefits of treating or opting not to treat anxiety and depression in the perinatal period. Current practice in this area emphasises risk-benefit analysis as an approach to dealing with the complexity of treatment decisions. However, ethical principles and legal frameworks may also provide some guidance for clinicians. A move to a system based on a presumption of capacity, supported decision-making and, when capacity is not present, relational ethics and substituted decisions that respect the patient's will and preferences, but in any case aim to maximise their welfare, would provide a scaffold that would enable clinicians and patients to work through this difficult area.

\section{Acknowledgements}

The helpful comments of Dr Christopher Ryan on early drafts of the manuscript are gratefully acknowledged.

\section{References}

American College of Obstetricians and Gynecologists (2008) At-risk drinking and illicit drug use: ethical issues in obstetric and gynecological practice. Committee Opinion No. 422. Obstetrics and Gynecology, 112 : 1449-60.

American College of Obstetricians and Gynecologists (2011) Substance abuse reporting and pregnancy: the role of the obstetrician-gynecologist. Committee Opinion No. 473. Obstetrics and Gynecology, 117: 200-1.

Anderson EL, Reti IM (2009) ECT in pregnancy: a review of the literature from 1941 to 2007. Psychosomatic Medicine, 71: 235-42.

Andersson L, Sundström-Poromaa I, Wulff M, et al (2004) Neonatal outcome following maternal antenatal depression and anxiety: a population-based study. American Journal of Epidemiology, 159: 872-81. 


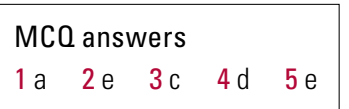

Beyondblue (2011) Clinical Practice Guidelines. Depression and Related Disorders - Anxiety, Bipolar Disorder and Puerperal Psychosis - in the Perinatal Period: A Guideline for Primary Care Health Professionals. Beyondblue.

Bledsoe SE, Grote NK (2006) Treating depression during pregnancy and the postpartum: a preliminary meta-analysis. Research on Social Work Practice, 16: 109-20.

Bogardus Jr ST, Holmboe E, Jekell JF (1999) Perils, pitfalls, and possibilities in talking about medical risk. JAMA, 281: 1037-41.

Brand SR, Brennan PA (2009) Impact of antenatal and postpartum maternal mental illness: how are the children? Clinical Obstetrics and Gynecology, 52: 441-55

Callaghan S, Ryan CJ (2012) Rising to the human rights challenge in compulsory treatment - new approaches to mental health law in Australia. Australian and New Zealand Journal of Psychiatry, 46: 611-20.

Chaudron LH (2013) Complex challenges in treating depression during pregnancy. American Journal of Psychiatry, 170: 12-20.

Coverdale JH, McCullough LB, Chervenak FA, et al (1997) Clinical implications of respect for autonomy in the psychiatric treatment of pregnant patients with depression. Psychiatric Services, 48: 209-12.

Cohen LS, Altshuler LL, Harlow BL, et al (2006) Relapse of major depression during pregnancy in women who maintain or discontinue antidepressant treatment. JAMA, 295: 499-507.

Deave T, Heron J, Evans J, et al (2008) The impact of maternal depression on early child development. British Journal of Obstetrics and Gynaecology, 115: 1043-51.

Dimidjian S, Goodman S (2009) Nonpharmacologic intervention and prevention strategies for depression during pregnancy and the postpartum. Clinical Obstetrics and Gynecology, 52: 498-515.

DiPietro JA, Novak MFSX, Costigan KA, et al (2006) Maternal psychological distress during pregnancy in relation to child development at age two. Child Development, 77: 573-87.

Dunkel Schetter C, Tanner L (2012) Anxiety, depression and stress in pregnancy: implications for mothers, children, research, and practice. Current Opinion in Psychiatry, 25: 141-8.

Galbally M, Snellen M, Lewis A (2011) A review of the use of psychotropic medication in pregnancy. Current Opinion in Obstetrics and Gynecology, 23: 408-14.

Gentile S, Galbally M (2011) Prenatal exposure to antidepressant medications and neurodevelopmental outcomes: a systematic review. Journal of Affective Disorders, 128: 1-9.

Goedhart G, Snijders AC, Hesselink AE, et al (2010) Maternal depressive symptoms in relation to perinatal mortality and morbidity: results from a large multi-ethnic cohort study. Psychosomatic Medicine, 72: 769-76.

Goodman JH (2009) Women's attitudes, preferences, and perceived barriers to treatment for perinatal depression. Birth, 36: 60-9.

Grote NK, Bridge JA, Gavin AR, et al (2010) A meta-analysis of depression during pregnancy and the risk of preterm birth, low birth weight, and intrauterine growth restriction. Archives of General Psychiatry, 67: 1012-24.

Halligan SL, Murray L, Martins C, et al (2007) Maternal depression and psychiatric outcomes in adolescent offspring: a 13-year longitudinal study. Journal of Affective Disorders, 97: 145-54.

Hays DF, Pawlby S, Waters CS, et al (2008) Antepartum and postpartum exposure to maternal depression: different effects on different adolescent outcomes. Journal of Child Psychology and Psychiatry, 49: 1079-88.

International Clearinghouse for Birth Defects Monitoring Systems (2003) Annual Report. ICBDMS.

Jensen HM, Grøn R, Lidegaard Ø, et al (2013) Maternal depression, antidepressant use in pregnancy and Apgar scores in infants. British Journal of Psychiatry, 202: 347-51.

Kordes-de Vaal JH (1996) Intention and the omission bias: omissions perceived as nondecisions. Acta Psychologica (Amsterdam), 93: 161-72.
Kurki T, Hiilesmaa V, Raitasalo R, et al (2000) Depression and anxiety in early pregnancy and risk for preeclampsia. Obstetrics and Gynecology, 95: 487-90.

Lyerly AD, Mitchell LM, Armstrong EM, et al (2009) Risk and the pregnant body. Hastings Center Report, 39: 34-42.

Marcus SM, Flynn HA, Blow FC, et al (2003) Depressive symptoms among pregnant women screened in obstetric settings. Journal of Women's Health, 12: 373-80.

Miller LJ (2009) Ethical issues in perinatal mental health. Psychiatric Clinics of North America, 32: 259-70.

National Institute for Health and Clinical Excellence (2007) Antenatal and Postnatal Mental Health: Clinical Management and Service Guidance (Clinical Guideline CG45). NICE.

Nulman I, Rovert J, Stewart, DE et al (1997) Neurodevelopment of children exposed in utero to antidepressant drugs. New England Journal of Medicine, 336: 258-62.

Nulman I, Rovet J, Stewart DE, et al (2002) Child development following exposure to tricyclic antidepressants or fluoxetine throughout fetal life: a prospective, controlled study. American Journal of Psychiatry, 159: 1889-95.

Oberlander TF, Warburton W, Misri S, et al (2006) Neonatal outcomes after prenatal exposure to selective serotonin reuptake inhibitor antidepressants and maternal depression using population-based linked health data. Archives of General Psychiatry, 63: 898-906.

Oberlander TF, Warburton W, Misri S, et al (2008) Effects of timing and duration of gestational exposure to serotonin reuptake inhibitor antidepressants: population-based study. British Journal of Psychiatry, 192: 338-43.

Pearlstein T (2008) Perinatal depression: treatment options and dilemmas. Journal of Psychiatry and Neuroscience, 33: 302-18.

Pitzer M, Jennen-Steinmetz C, Esser G, et al (2011) Prediction of preadolescent depressive symptoms from child temperament, maternal distress, and gender: results of a prospective, longitudinal study. Journal of Developmental and Behavioural Pediatrics, 32: 18-26.

Punamäki R-L, Repokari L, Vilska S, et al (2006) Maternal mental health and medical predictors of infant developmental and health problems from pregnancy to one year: does former infertility matter? Infant Behaviour and Development, 29: 230-42.

Rai D, Lee BK, Dalman C, et al (2013) Parental depression, maternal antidepressant use during pregnancy, and risk of autism spectrum disorders: population based case-control study. BMJ, 346: f2059.

Ramos E, Oraichi D, Rey E, et al (2007) Prevalence and predictors of antidepressant use in a cohort of pregnant women. British Journal of Obstetrics and Gynaecology, 114: 1055-64.

Reck C, Zimmer K, Dubber S, et al (2013) The influence of general anxiety and childbirth-specific anxiety on birth outcome. Archives of Women's Mental Health, 16: 363-9.

Ross LE, McLean LM (2006) Anxiety disorders during pregnancy and the postpartum period: a systematic review. Journal of Clinical Psychiatry, 67: 1285-98.

Ryding EL, Wijma B, Wijma K, et al (1998) Fear of childbirth during pregnancy may increase the risk of emergency cesarean section. Acta Obstetrica Gynecologica Scandinavica 77: 542-7.

Sanz E, Gómez-López T, Martínez-Quintas MJ (2001) Perception of teratogenic risk of common medicines. European Journal of Obstetrics, Gynecology and Reproductive Biology, 95: 127-31.

Scottish Intercollegiate Guidelines Network (2002) Postnatal Depression and Puerperal Psychosis. Section 4: Prescribing Issues in Pregnancy and Lactation. SIGN. Available at: http://www.sign.ac.uk/guidelines/ fulltext/60/section 4 html.

Seeman MV (2004) Relational ethics: when mothers suffer from psychosis. Archives of Women's Mental Health, 7: 201-10.

Seng JS, Oakley DJ, Sampselle CM, et al (2001) Posttraumatic stress disorder and pregnancy complications. Obstetrics and Gynecology, 97: $17-22$. 
Stephansson 0, Kieler H, Haglund B, et al (2013) Selective serotonin reuptake inhibitors during pregnancy and risk of stillbirth and infant mortality. JAMA, 309: 48-54.

Suri R, Altshuler L, Hellemann G, et al (2007) Effects of antenatal depression and antidepressant treatment on gestational age at birth and risk of preterm birth. American Journal of Psychiatry, 164: 1206-13.

Wisner KL, Zarin DA, Holmboe ES, et al (2000) Risk-benefit decisionmaking for treatment of depression during pregnancy. American Journal of Psychiatry, 157: 1933-40.

Wisner KL, Sit DK, Hanusa BH, et al (2009) Major depression and antidepressant treatment: impact on pregnancy and neonatal outcomes. American Journal of Psychiatry, 166: 557-66.
Yonkers KA, Wisner KL, Stewart DE, et al (2009) The management of depression during pregnancy: a report from the American Psychiatric Association and the American College of Obstetricians and Gynecologists. General Hospital Psychiatry, 31: 403-13.

Ververs T, Kaasenbrood H, Visser G, et al (2006) Prevalence and patterns of antidepressant drug use during pregnancy. European Journal of Clinical Pharmacology, 62: 863-70.

Zuckerman B, Amaro H, Bauchner H, et al (1989) Depressive symptoms during pregnancy: relationship to poor health behaviors. American Journal of Obstetrics and Gynecology, 160: 1107-11.

\section{$R$ v Iby [2005] NSWCCA 178.}

St George's Healthcare NHS Trust $v$ S; $R$ v Collins and others, ex parte $S$ [1998] 3 All ER 673.

\section{MCQs}

Select the single best option for each question stem

1 Of the following, the most consistent evidence of an adverse association with SSRIs in the perinatal period is seen in:

a serotonin discontinuation syndrome in neonates

b spontaneous abortion

c prematurity

d pre-eclampsia

e low birth weight.

2 In discussing the treatment of antenatal depression with pregnant women, the clinician should cover:

a background risk of malformations

b impact of untreated depression on fetal development

c potential adverse effects of SSRIs on neonatal outcomes

d psychotherapy

e all of the above.

\section{Pregnancy-related anxiety is:}

a not associated with any adverse obstetric outcomes

b normal

c more consistently associated with adverse obstetric and neonatal outcomes than panic disorder

d fear of giving birth

e best treated with benzodiazepines in combination with SSRIs.

4 A 42-year-old woman is 20 weeks pregnant with her first baby via IVF after 3 years of infertility treatment. She presents with anhedonia, poor weight gain, insomnia, depressed mood and ruminating thoughts of being unable to care for her baby. She declines antidepressants and is not interested in psychotherapy. The least important factor in making decisions about her treatment is:

a evaluating the influence of her depressive symptoms on decision-making capacity b the perspective of her husband on her premorbid self

c suicidal ideas

d ascertaining whether she is still able to work

e the risks of taking antidepressants in pregnancy.

5 A 30 -year-old mother of two children (ages 5 and 2) presents in the third trimester of her third planned pregnancy with obsessional thoughts of harming her fetus, catastrophic thoughts regarding the outcome of the coming birth, panic attacks, insomnia and inability to emotionally attend to her children. The most useful framework for making decisions about treatment of her anxiety is:

a the child protection system

b the Mental Health Act

c risk-benefit analysis

d societal conventions

e relational ethics. 